\title{
Study on Service Quality of Chain Pharmacy-a Case Study of Chain Pharmacy in Guangdong Province
}

\begin{abstract}
Zhang Yu
Macau University of Science and Technology, Macau, China

enlong0214@163.com

Abstract

In the new medical reform plan, it is proposed to establish and improve the drug supply security system, which makes the people's demand for medicine gradually expand, bringing huge development opportunities to the drug chain enterprises. Therefore, the improvement of service quality of chain pharmacies is particularly important for improving customer loyalty and thus for pharmacies to benefit from satisfying customer demand. Using expert scale of chain pharmacy service quality evaluation, finally used multiple regression equation to explain chain pharmacy service quality and the relationship between the customer loyalties to the pharmacy, and further discusses how different dimensions affects customer loyalty, and connecting with the data analysis results of chain pharmacy service quality promotion strategy.
\end{abstract}

Keywords: chain pharmacy, service quality, customer loyalty

\section{连锁药房服务质量研究一以广东地区连锁药房为例}

\author{
张宇
}

澳门科技大学澳门中国

enlong0214@163.com

\begin{abstract}
摘要
在新的医改方案中, 提出建立健全药品供应保障体系, 这使得老百姓对医药需求逐步扩大, 给药品连 锁企业带来了巨大的发展机遇。因此，连锁药房服务质量的提升对于提高顾客忠诚度，进而药房从满 足顾客需求中获利是尤为重要的。运用专家量表对连锁药房服务质量进行测评, 最终运用多元回归方 程解释连锁药房服务质量与顾客对药房忠诚度之间的关系, 并进一步探讨不同维度是如何影响顾客忠 诚的, 并结合数据分析结果提出对连锁药房服务质量提升的策略。
\end{abstract}

关键词: 连锁药房, 服务质量, 顾客忠诚

\section{1. 引言}

目前, 中国国内对于连锁药房尚无统一的定义，本 人在走访部分药学专家和珠海市医药流通行业协会部 分商家成员，定义为在一个医药企业统一部署之下，管 理着有共同经济利益的众多药房, 以授权、特许经营等 方式集合起来, 共享经济效益的一种组织形式 [1]。根据 《2015 中国连锁药房行业研究报告》显示至今, 国内的 连锁药房发展已 20 余年; 但随着连锁药房数量的不断 增加, 这个市场开始逐步被媒体曝光问题。在这个意义 上讲, 顾客通过服务质量对连锁药房的忠诚度高低, 直 接影响着药房企业的利润和发展, 因此研究连锁药房的 服务质量与顾客忠诚之间的关系是非常之必要的。
本文选取广东三个比较有代表性的城市中比较有 影响力的三个连锁药房，他们分别是：广州市宝芝林大 药房连锁有限公司成立于 2000 年 10 月，总部位于广州 市越秀区。本着质量求信誉, 信誉求发展的理念, 公司 和门房成为广州市最早实行和通过 GSP 认证的企业之一; 深圳中联大药房 1995 年以来, 中联大药房曾荣获 “全国 医药商品质量管理规范单位” 等光荣称号。2003 年一次 性通过国家 “GSP” 认证, 2005 年进入中国连锁百强。 同时连续六年入围中国连锁药房百强榜前列; 珠海市嘉 宝华健康药房连锁股份有限公司，成立于 2007 年，是一 家集医药制造、批发、零售为一体的集团化企业。公司 注册资本 12100 万元，年营业规模 3 亿余元，拥有员工 近 1000 人, 位列全国连锁药房综合实力和综合竞争力 百强企业。 


\section{2. 相关理论文献综述}

\section{1 服务质量的相关研究}

\subsection{1服务定义}

在 1960 年, 美国市场营销协会对其有这样的定义, 服务是指出售、或是与产品连带出售的活动、利益亦或 是满足感。在国内也有学者, 将其按不同类别进行界定, 在总结国内外文献研究认为: 第一类是从过程角度加以 界定;第二类是从服务的内在特性加以界定;第三类是 与实体产品的对比中加以界定。综合以上定义, 在本次 研究中, 认为连锁药房提供给顾客的服务分为两类: 一 类是提供给顾客的有形产品服务即药品; 二类是在提供 药品服务时顾客自身所能感知的人文关怀和说明等无 形产品。

\subsection{2 服务质量定义}

服务质量它是某种测量企业服务水平是否满足顾 客期望程度的工具 [2]。在这之后, 又有学者在研究服务 质量时认为它一种态度, 即服务质量是衡量消费者本身 对服务的期望和消费者接受服务后实际感受到服务效 果的知觉 [3]。我国国内的研究一般针对不同服务行业 质量评价的方法: 如有学者对中国大陆的宾馆服务质量 研究时, 发现无形服务的质量对顾客满意程度有很大的 影响 [4]。

本次研究对象为连锁药房, 基于药品这种特殊商品, 认为其服务质量应该满足于以下几个方面: 1) 提供药品 服务的安全性, 保障顾客的用药安全是药房第一遵守的 行业准则; 2) 以顾客为出发点, 满足其用药需求; 3) 与顾客建立有效的沟通和倾听机制, 对顾客忠诚度不定 期进行评测与研究。

\subsection{3 服务质量 SERVQUAL 模型}

Parasuraman、Zeitham1 和 Berry 三位学者, 综合 前人研究的成果, 选择当时比较普遍的服务性产业, 即 银行和信用卡公司以及证券经纪商和维修厂这四类进 行探索性实证研究, 最后提出服务质量有五个差距缺口。 并在 1988 年和 1991 年通过阶段性的实证研究和量表修 正后, 把五个缺口演变为服务质量的五个维度, 即有形 性、可靠性、响应性、保证性和移情性, 并以 22 道具有 良好效度与信度的题目去衡量服务质量的量表, 即随后 被广泛使用的 SERVQUAL 模型。

\section{1.4 连锁药房的服务质量模型}

2006 年, 专家蒋利林和杨世民提出可以按照实体、
可靠、关怀、反应和问题解决这五类指标评测零售连锁 药房服务质量 [5]; 2009 年, 学者钟素艳和李玲认为可 以从基础服务、药学服务、信息服务、关怀性服务这四 个方面着手, 对服务质量进行测评 [6]。直到在 2010 年, 倪永兵等以大量的零售药房样本作为研究对象, 通过 $\mathrm{EFA}$ 分析, 提出连锁药房服务质量可以划分为: 服务的 周到性、服务能力、可信性、服务环境以及便利性五个 维度, 最后通过 CFA 分析并对进行修正，修正后模型拟 合度良好, 信度和效度较高, 可用于国内零售药房服务 质量的评测 [7]。因此, 本次连锁药房的服务质量的评测 用这个专家量表。

\section{2 顾客忠诚的相关研究}

\subsection{1 顾客忠诚的定义}

这里主要是指在顾客购买这种行为的重复性。对于 重复购买的次数, 美国部分营销专家认为是 3 至 4 次重 复购买。一些中国大陆的学者也对此进行了研究: 如通 过研究认为顾客忠诚度是由质量、价格、服务等多个方 面共同作用的结果 [8]。在本次研究中, 连锁药房在顾客 忠诚方面应该以满足消费者（具体是指到门房内购买药 品的顾客）的服务需求，即当顾客在消费时或之后，其 核心的服务利益得到满足后, 进而才能提高顾客对本连 锁药房的忠诚度。

\subsection{2 顾客忠诚的衡量方法}

顾客忠诚有许多的衡量方法，我们大致可以分为三 种。一种是用顾客行为来衡量, 即认为可以即顾客的购 买频率、数量等行为进行分析 [9]; 第二种是顾客的态度 上衡量, 即有学者认为可以从顾客口碑、推荐等态度来 衡量 [10]; ; 第三种是组合方式来测量, 即有学者提出合 成方法, 即把态度和行为测量组合在一起 [11]。本文对 连锁药房的顾客忠诚度测量也将采用第三种方式。

\subsection{3 服务质量与顾客忠诚}

关于他们之间的关系，有两种观点：第一种是认为 服务质量对顾客忠诚有着直接影响, 在 1996 年, Zeithaml、Berry 和 Parasuraman 三位学者通过对顾客 与企业关系的行为研究中, 发现了这种直接影响; 第二 种是认为二者没有明显的直接影响, 而是间接的影响顾 客忠诚: 有学者经研究发现如果企业能提高对顾客的满 意度将间接影响顾客忠诚 [12]。在国内, 有学者在实证 研究中发现, 服务质量直接影响着顾客忠诚的程度 [13]。 本次研究认同第一类观点, 将连锁药房的服务质量当作 一个整体变量, 试图和顾客忠诚建立联系, 并研究服务 质量的各个要素与顾客忠诚的影响。 
同

H2. 3: 不同学历顾客对连锁药房的顾客忠诚有所不 同

H2. 4: 不同个人月收入顾客对连锁药房的顾客忠诚 有所不同

\section{2 问卷设计与数据收集}

\subsection{1 问卷评测构成说明}

本次问卷分为两个部分，即第一部分是对连锁药房 服务质量和顾客忠诚的问题，另一部分是关于人口特征 变量的调查问题; 在顾客回答服务质量问题时, 采用 Likert 量表 5 级来评测, 即完全不同意, 并赋值 1 来表 示; 不同意, 并赋值 2 来表示; 一般, 并赋值 3 来表示; 同意, 并赋值 4 来表示; 完全同意, 并赋值 5 来表示。

问卷中服务质量测量问题包括五个方面，服务的周 到性: 建立顾客药历（过往购买药品的经历）、定期发放 一些安全用药的健康咨询、顾客回访、开展药学服务宣 传、提供相应的药学服务书籍; 服务的能力: 及时更正 服务过程中的失误和差错、员工有丰富的药学常识、给 予顾客购买药品的专业指导、员工依据病情推荐给顾客 适合的药品、员工对处方药品的正确调配、员工对顾客 问题迅速做出回答、及时处理顾客投诉; 服务的可信性: 有区分处方和非处方药品的警示标语、药品的摆放及合 理区分、员工医学背景和药品经营许可的公示、提供质 量合格的药品、药房内设有医药监管部门的投诉或举报 电话; 服务环境: 药房环境干净舒适、员工着装整洁举 止文明、药房内有便民设施; 服务的便利性：药品种类 丰富且充足、药房能 24 小时运营、有开展方便顾客服务 (如免费测血压、体温等)。在顾客忠诚问题设置有: 以 后会向他人推荐此药房、以后会再次光顾此药房、以后 会关注此药房的发展，共计三个问题。

\subsection{2 问卷资料的收集}

本次研究是针对广东省连锁药房为调查对象, 所收 集的样本, 均来自进入连锁药房消费药品服务的顾客, 所以采用的判断抽样方法。通过两种发放途径进行：一 是对进入连锁药房消费药品的顾客, 一线员工一对一进 行问卷调查; 第二种途径是, 通过新媒体方式即微信朋 友圈回收问卷。微信朋友圈问卷有一个前提问题设置: 是否在本药房购买过药品; 如回答否, 则终止答卷。

\section{4. 研究结果}

问卷共计发出 350 份（含微信朋友圈调查问卷 100 份）调查问卷, 共计回收 317 (含微信朋友圈调查问卷 50 份）份问卷，回收问卷率为 $91 \%$ 。为了使本次研究更 为可靠, 将有缺失值等问题的无效问卷进行剔除, 最终

H2. 2: 不同年龄顾客对连锁药房的顾客忠诚有所不 


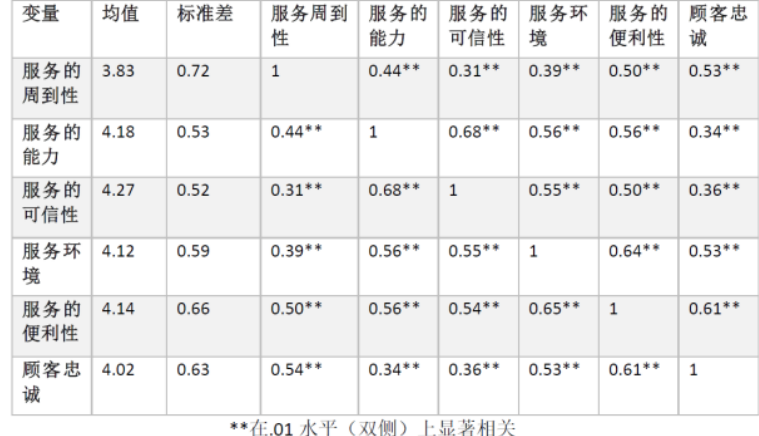

其中，服务的便利性与顾客忠诚之间的 Pearson 相关系数 (0.61) 最大, 服务的周到性和服务环境分别 与顾客忠诚度之间的 Pearson 相关系数 (0.54 和 0.53$)$ 次之。

\section{4 回归分析}

本文将构建如下多元回归模型: $\mathrm{Y}=\mathrm{aX}_{1}+\mathrm{bX}_{2}+\mathrm{cX} \mathrm{X}_{3}+$ $\mathrm{dX}_{4}+\mathrm{e} \mathrm{X}_{5}+\mathrm{f}$ ( $\mathrm{Y}$ : 顾客忠诚 (因变数); $\mathrm{X}_{1}$ : 周到性; $\mathrm{X}_{2}$ : 服务能力; $X_{3}$ : 可信性; $X_{4}$ : 服务环境; $X_{5}$ : 便利性 $\left(X_{1}-\right.$ $X_{5}$ 为自变量); $f$ : 常数); 对模型的方差分析结果显示 $\mathrm{P}$ 值 $<0.01$, 说明此模型可以继续做回归分析。方差分析 见下表:

\begin{tabular}{|l|l|l|l|l|}
\hline 模型 & 平方和 & df & F & P \\
\hline 回归 & 58.517 & 5 & 54.575 & 0.000 \\
\hline
\end{tabular}

现进行多元回归分析, 结果如下: 信。上五个维度的测量结果显示 $\alpha$ 系数都大于 0.7 , 说明可信度很高, 并且问卷整体的 a 系数为 0.920 , 标 准属于十分可信。综上所述, 问卷整体及内部各构建层 面可信度较高, 符合进行研究分析的标准。

在内容效度方面, 本文选用 Parasuraman、Zeitham1 和 Berry 三位学者在 1988 提出的经典模型---SERVQUAL 模型, 作为量表的分析基础模型; 同时经查阅文献, 选 用国内 2010 年倪永兵 (博士)、褚淑贞 (副教授) 和黄 文龙 (教授、博士生导师) 三位学者提出的连锁药房服 务质量评测量表。综合以上, 有理由相信本次研究是在 专家学者之判断基础之上的, 故具有很好的内容效度。 在结构效度方面, 本文采用的专家量表, 即上文提到的 倪永兵 (博士)、褚淑贞 (副教授) 和黄文龙（教授、博 士生导师）三位学者已经在研究时通过验证性因子分析, 故具有很好的结构效度。

\section{3 相关性分析}

本次研究对连锁药房服务质量的五个维度, 进行 Pearson 相关系数分析。分析结果见下图:

\begin{tabular}{|c|c|c|c|c|c|c|}
\hline 变量 & B & 标准误差 & \multicolumn{2}{|c|}{ 标准系数 } & $\mathbf{t}$ & $\mathbf{P}$ \\
\hline 服务周到性 & 0.284 & 0.044 & \multicolumn{2}{|c|}{0.323} & 6.485 & 0.000 \\
\hline 服务能力 & -0.195 & 0.074 & \multicolumn{2}{|c|}{-0.165} & -2.647 & 0.009 \\
\hline 服务可信性 & 0.055 & 0.073 & \multicolumn{2}{|c|}{0.045} & 0.750 & 0.455 \\
\hline 服务环境 & 0.258 & 0.063 & \multicolumn{2}{|l|}{0.241} & 4.081 & 0.000 \\
\hline 服务便利性 & 0.346 & 0.059 & \multicolumn{2}{|l|}{0.361} & 5.911 & 0.000 \\
\hline $\mathbf{R}$ 方 & 调整 R 方 & \multicolumn{2}{|c|}{ 标准估计误差 } & \multicolumn{2}{|c|}{$\mathbf{R}$ 方更改 } & F 更改 \\
\hline 0.481 & 0.473 & 0.46308 & & \multicolumn{2}{|c|}{0.481} & 54.575 \\
\hline
\end{tabular}

基于以上两个表格, 得知: 其调整后的判定系数即 R2 为 0.473 (在 0-1 之间), 可以解释回归方程; 其中 服务的可信性的 $\mathrm{P}$ 值 $>0.05$, 其他维度的 P 值都小于 0.05 , 这四个变量可以进入多元回归方程。通过以上, 我 们可以得知在连锁药房服务质量五个维度中, 除服务的 可信性以外, 其他维度都与顾客忠诚存在着或正或负的 直接影响; 对于之前所涉及的假设, H1 部分成立, H1. 3 不成立，其余 H1. 1、H1.2、H1. 4、H1. 5 成立。

\section{5 顾客属性分析}

首先，进行独立样本 $\mathrm{T}$ 检验来分析假设 H2. 1 ; 其结 果 P 值 0.439 大于 0.05 , 所以不存在显著差异, 因此顾 客性别对于忠诚度没有影响, 假设 H2. 1 不成立; 将顾客 不同年龄层次、不同学历层次、不同月收入层次都进行 ANOVA 分析, 其 P 值 0.000 都小于 0.05 , 有显著差异, 因此假设 H2. 2、H2.3、H2. 4 都成立。 
病情推荐合适药品的能力和药品知识掌握能力等。3、对

\section{5. 结论与建议}

\section{1 相关研究结论}

经过问卷调查与统计分析, 得出连锁药房之服务质 量的五个评测变量--周到性、服务能力、服务可信性、 服务环境、便利性之间存在显著的相关性。并在相关强 弱性上说明，便利性、周到性和服务环境这三个维度与 顾客忠诚度的相关性比较于服务的能力和服务的可信 性更强。经实际数据测算, 之前所涉及的研究假设是否 成立, 如下表:

\begin{tabular}{|c|c|}
\hline 所涉及研究假设内容 & 结果 \\
\hline 连锁药店的服务质量对顾客忠诚有正向影响 & 部分成立 \\
\hline 连锁药店的服务周到性对顾客忠诚有正向影响 & 成立 \\
\hline 连锁药店的服务能力对顾客忠诚有正向影响 & 成立 \\
\hline 连锁药店的服务可信性对顾客忠诚有正向影响 & 不成立 \\
\hline 连锁药店的服务环境对顾客忠诚有正向影响 & 成立 \\
\hline 连锁药店的服务便利性对顾客忠诚有正向影响 & 成立 \\
\hline 不同顾客的属性对连锁药店的顾客忠诚有所不同 & 部分成立 \\
\hline 不同性别顾客对连锁药店的顾客忠诚有所不同 & 不成立 \\
\hline 不同年龄顾客对连锁药店的顾客忠诚有所不同 & 成立 \\
\hline 不同学历顾客对连锁药店的顾客忠诚有所不同 & 成立 \\
\hline $\begin{array}{l}\text { 不同个人月收入顾客对连锁药店的顾客忠诚有所 } \\
\text { 不同 }\end{array}$ & 成立 \\
\hline
\end{tabular}

\section{2 相关管理实践启示}

在本次研究中，被调查的 300 位样本顾客中，其不 同年龄层次、不同学历背景和不同收入类型对顾客忠诚 有差异化表现。举例来说, 本次研究中学历层次在大学 本科和研究生学历中对顾客忠诚度的均值分别为 3.84 、 3.72 , 比高中至大专学历的略低, 表明这个学历层次群 体对忠诚度有更高的要求, 也就是说对其服务质量的要 求也比较高; 所以在药房对待这些顾客进行一对一服务 时, 一线作人员要尽最大可能满足其购买需要, 避免盲 目或硬性推销, 用专业推荐代替顾客的被动选择, 这样 才能培养其忠诚度, 进而再次光顾本药房。

本文从实证研究出发, 通过多元回归分析, 证实本 次服务质量有四个维度 (周到性、服务能力、服务环境、 便利性) 对顾客忠诚有显著影响; 所以连锁药房需要从 这四个维度做起提高服务质量, 进而培养其顾客的忠诚 度。1、对于服务的周到性来讲, 可以把重点放在教育顾 客相关药学知识的层面上来进行。顾客进入药房实际上 是因为病痛而购买药品, 其实也是在 “消费” 健康; 但 是据某些媒体报导存在滥用药物的情况非常之多。从顾 客角度出发, 不定期推出健康咨询或书籍并免费发放; 建立会员制度, 对其进行电话回访, 及时沟通病情进展 或是用药情况等。2、对于服务的能力上来讲, 可以把重 点放在服务接触这个层面上来进行。在学术界, 多数学 者认为服务接触是顾客感知的基础, 这种接触瞬间是愉 快的或是令人满意的, 亦或是令人沮丧的等, 都会影响 顾客忠诚。如提高及时处理顾客投诉的能力、依据顾客
于服务环境和便利性上来讲，可以把重点放在发挥服务 场景的战略作用上来进行。如在药房中, 提供一些便民 设施和服务。举例来说, 如果一位老人顾客在购买药品 时能够坐在椅子上接受咨询或是购买行为, 该顾客将会 非常高兴，继而会促使其再次光顾药房进行再次消费。

\section{3 研究限制}

本次研究虽然查阅了国内外专家学者的文献, 并通 过严肃的资料分析得到上述结果，但因各方面的因素存 在一定的限制, 主要是以下两个方面: 1、地区及研究对 象限制: 在本次研究中, 仅选取三所代表性城市的连锁 药房所以对其服务质量研究受到限制，不具有普遍性。 2、问卷样本的限制: 本次研究中, 选取样本的时间和, 区域都比较集中; 而且采取的是抽样判断方法, 所以在 回收问卷所代表的数据存在一定的局限。

本文所研究实际是涉及到服务营销这门学科, 涉及 管理、统计、心理等方面的学科背景影响; 针对本次的 研究对象提出以下未来的研究方面: 1、拓展研究范围, 可以从广东省扩展至全国, 开发适合不同区域的普遍量 表。2、本次研究中, 只是涉及了服务质量与顾客忠诚二 者之间的关系，并没有考虑学术界对于中介变量或是调 节变量的考虑, 如顾客价值、顾客满意度、顾客行为或 是态度等。今后将引入这些, 作进一步研究。

\section{REFERENCES}

[1] Ni Yongbing. (2013) reflections on the establishment of drug safety management regulations in drug retail chain enterprises [J]. China pharmacy (24) : 4229-4230

[2] Lewis R C, Booms B H. ( 1983 ) The marketing aspects of service quality in emerging perspectives on services marketing. In: Berry L, Shostack G, Upah G ed. Chicago: American Marketing: 99- 107.

[3] Parasuraman A., V. A. Zeithaml, \& L. L. Berry. (1988). SERVQUAL: A multiple- item scale for measuring consumer perceptions of service quality. Journal of Retailing, 64(No.1, Spring), 12-40

[4] Wang Chunxiao et al. (1996) empirical research on service quality attributes $[\mathrm{J}]$. Business research. (6) 12-14

[5] Jiang Lilin, Yang Shimin (2006) to establish a service quality evaluation model for retail chain pharmacies $[\mathrm{J}]$. China pharmacies. (9): 718-719

[6] Zhong Suyan, Li Lingyu et al. (2009) study on service quality evaluation system of retail pharmacies [J]. China Pharmacoeconomics. (3): 112-20

[7] Ni Yongbing, Chu Shuzhen. (2010) an empirical study 
on quality evaluation of pharmaceutical care in retail pharmacies based on factor analysis [J]. Nanjing social sciences. J: 41-46

[8] Zheng Xiaoyun. The construction process of customer loyalty in the development of budget hotels [J]. Modern marketing (academic edition), 2012 (06): 20

[9] Jones T O, Sasser W E. Why Satisfied Customers Defect[J]. Harvard Business Review, 1995(6): 88-99.

[10] Reichheld F F.The Loyalty Effect[M].Boston: Harvard Business School Press,1996: 19-21.
[11] Chen Y, Xie J. Cross-market Network Effect with Asymmetric Customer Loyalty: Implications for Competitive Advantage[J].Marketing Science, 2007(1): 5266.

[12] Susan M. Keaveney. Customer Switching Behavior in Service Industries: An Exploratory Study. 1995, 59(2):71-82.

[13] Song Chunhong, Su Jingqin. Empirical test on the influence of service quality, customer value and customer satisfaction on customer loyalty [J]. Statistics \& decision, 2008 (19): 182-184 